\title{
Semen parameters and electron microscope observations of spermatozoa of the red wolf, Canis rufus
}

\author{
J. K. Koehler ${ }^{1}$, C. C. Platz, Jr ${ }^{2}$, W. Waddell ${ }^{3}$, M. H. Jones ${ }^{4}$ and S. Behrns ${ }^{3}$ \\ ${ }^{1}$ University of Washington, School of Medicine, Seattle, WA 98195, USA; ${ }^{2}$ International Canine Semen Bank, Sandy, OR 97055, USA; \\ ${ }^{3}$ Point Defiance Zoo and Aquarium, Tacoma, WA 98407, USA; and ${ }^{4} J o n e s$ Animal Hospital, Tacoma, WA 98409, USA
}

\begin{abstract}
Semen parameters were evaluated on ejaculates of a captive population of red wolves (Canis rufus) sampled over two consecutive mating seasons. A total of 31 samples from 15 animals yielded mean sperm motility of $69.6 \pm 19.4 \%$, mean sperm density of $131 \pm$ $124 \times 10^{6} \mathrm{ml}^{-1}$, mean total number of spermatozoa of $470 \pm 465 \times 10^{6}$ and mean percentage morphologically abnormal spermatozoa of $35 \pm 11.8 \%$. Restricting the data to animals sampled three times or more or limiting the samples to proven breeders resulted in statistically non-significant differences in these numbers $(P<0.05)$. When compared with data from other canines the seminal parameters of red wolves are at the lower extremes of the range. In particular the proportion of morphologically abnormal spermatozoa $(35 \%)$ is approximately twice that seen in other canine species. Light microscopic analysis of abnormal forms revealed that almost half $(45 \%)$ were bent defects, another $40 \%$ were secondary defects (coiled, detached and immature) and $15 \%$ were primary defects. Electron microscopy confirmed the presence of substantial numbers of morphologically abnormal forms including double-headed and doubleflagellar cells, bent or kinked forms especially in the neck region, acrosomal abnormalities and bizarre spermatids. Approximately one-third of the samples also showed the presence of white blood cells, in some cases demonstrating sperm phagocytosis (spermophagy). These results are consistent with the concept of declining sperm parameters associated with restricted gene pools in numerically limited populations. However, alternative explanations are also explored.
\end{abstract}

\section{Introduction}

Red wolves ranged widely over the south-eastern United States westward to Texas and into northern Mexico. A combination of man-made circumstances, including the devastation of the red wolf population, as well as its habitat, in the first half of the twentieth century, led to a steady numerical decline in the species (Shaw and Jordan, 1977). The red wolf was designated an endangered species in 1967 and by 1980 was declared to be extinct in the wild (US Fish and Wildlife Service, 1990). A red wolf recovery plan initiated by the US Fish and Wildlife Service has fostered an extensive effort to enhance this population through augmented reproductive strategies such as artificial insemination with the small numbers of animals still available in zoos and captive breeding facilities. Approximately 130 animals were accounted for in the initial survey (US Fish and Wildlife Service, 1990). Efforts that included natural and augmented reproductive stategies increased the census to 289 animals in 1995 (Waddell and Behrns, 1996).

Despite the fact that dog spermatozoa were among Leeuwenhoek's early depictions of microscopic observations

Received 18 November 1997. in 1679 , there is still much to learn about the factors that affect the semen parameters and sperm structural characteristics of domestic dogs and their relationship to fertility (Oettle, 1993). Furthermore, there is only limited information on wild canids. An earlier study of red wolf spermatozoa (Koehler et al., 1994) reported that extensive pyospermia and spermophagy was present in semen samples collected from red wolves. In an extension of that work, the present paper reports semen parameters and electron microscope observations on specimens collected during the breeding seasons of 1994 and 1995, with special emphasis on abnormal spermatozoa. The aim is to present data that may be compared with other canines and help to form a baseline of information on red wolves that may be useful to optimize sperm quality and improve fertilization rates.

\section{Materials and Methods}

\section{Semen collection and analysis}

Samples used in this study were collected during the winter and spring breeding seasons of 1994 and 1995 at the Captive Breeding Facility of the Point Defiance Zoo located 
at Graham, WA, USA. Animals were transferred a short distance from their large open pens to a collection room and anaesthetized with an intramuscular injection of $175 \mathrm{mg}$ Telazol (tiletamine hydrochloride and zolazepam hydrochloride, Parke-Davis/Warner Lambert Co., Morris Plains, NJ). Semen was collected by electroejaculation using a P-T Electronics Model 302 and No. $4(1.6 \mathrm{~cm}$ diameter) rectal probe according to the method of Platz and Seager (1978).

After carefully cleaning the penis, the bladder was catheterized and urine was removed as completely as possible. Semen was collected in small beakers during each of three sets of 35-40 stimulations with 5 min rest intervals. Unfractionated semen samples were pooled, unless obviously contaminated with urine, and evaluated microscopically with phase contrast at $\times 400$ magnification

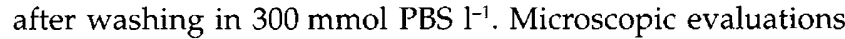
were performed immediately upon collection of semen and diluent solutions maintained at $37^{\circ} \mathrm{C}$. The parameters scored included percentage motility, concentration (density) and percentage of morphologically abnormal spermatozoa (categorized as primary, secondary or tertiary abnormalities). The presence of white or red cells was estimated by examining a 10-fold dilution of fixed cells at $\times 400$ in phase contrast. Means, standard deviations and $t$ tests of quantitative data were obtained using the Minitab 8 statistical software with a Macintosh LCIII computer.

\section{Electron microscopy}

Small aliquots of PBS-washed ejaculates were processed for electron microscopy according to the methods of Koehler et al. (1994). Briefly, fixation in $0.1 \mathrm{~mol}$ cacodylate $\mathrm{l}^{-1}$ buffered $1.25 \%(\mathrm{v} / \mathrm{v})$ glutaraldehyde was followed by $1 \%(\mathrm{w} / \mathrm{v})$ osmication, ethanol-propylene hydroxide dehydration and embedding in Epon 812. Thin sections cut with a diamond knife-equipped Sorvall PB-II ultramicrotome were stained with uranyl acetate and lead citrate and viewed in a Philips 420 electron microscope.

\section{Results}

\section{Semen analysis}

In addition to the summary data from the semen analysis presented in Table 1, five of the animals were sampled in both 1994 and 1995 and six specimens showed motility of less than $50 \%$. Ten of the 31 samples showed evidence of white blood cells ranging from two to 20 cells per $\times 400$ field and five showed red blood cells ranging from one to 20 cells per $\times 400$ field. Owing to time and facility constraints associated with the field conditions, only about half of the samples were evaluated for the presence of white cells, red cells or spermatocytes, so it was decided that quantitation of the blood cell data to a higher degree was not warranted.

A subset of the data was constructed based on multiple ejaculations, in which mean figures were calculated for five wolves, each of which was sampled three times or more (Table 1). Although the semen parameters are similar to the overall data, the standard deviations and ranges are slightly smaller for this selective subgroup.

On the supposition that known breeders exhibit a higher or more consistent set of semen parameters, a further division of data can be made by selecting only animals of known fertility. Seven of the 16 animals included in the study were known breeders and provided 16 of the 31 specimens collected. The mean values of this group were not significantly different from the population taken as a whole. A standard $t$ test applied to the three sets of data (Table 1) indicated no significant differences $(P<0.05)$.

Sperm abnormalities were scored using phase-contrast light microscopy $(\times 400)$ at the time of collection. Table 2 summarizes the data collected from the 31 ejaculates that were also processed for electron microscopy. Categories of primary abnormalities other than those listed were included in the original scoring, but only those with scores $>0$ are presented (Table 2). Almost half (45\%) of the abnormal number of spermatozoa was due to bent spermatozoa, $40 \%$ were equally distributed among the secondary abnormality categories, with primary (developmental) abnormalities contributing approximately $15 \%$ to the total.

Table 1. Mean semen parameters for red wolves

\begin{tabular}{lllll}
\hline & $\begin{array}{c}\text { Number of } \\
\text { Percentage motility } \\
\text { of spermatozoa }\end{array}$ & $\begin{array}{c}\text { Total number of } \\
\text { spermatozoa } \\
\left(\times 10^{6} \mathrm{ml}^{-1}\right)\end{array}$ & $\begin{array}{c}\text { Percentage abnormal } \\
\text { spermatozoa }\end{array}$ \\
\hline A & $69.6 \pm 19.4(10-95)$ & $131 \pm 124(11-560)$ & $470 \pm 465(0-2035)$ & $35.0 \pm 11.8(13-58)$ \\
B & $76.3 \pm 9.0(10-95)$ & $110 \pm 67(32-264)$ & $397 \pm 356(0-1240)$ & $34.0 \pm 3.8(28-52)$ \\
C & $66.9 \pm 26.0(10-95)$ & $125 \pm 133(11-560)$ & $467 \pm 442(0-1380)$ & $33.0 \pm 10.7(13-52)$
\end{tabular}

A: mean values ( \pm SD) for all samples ( 31 specimens from 15 wolves).

$B$ : mean values $( \pm$ SD) for five animals sampled three times or more.

$\mathrm{C}$ : mean values ( $\pm \mathrm{SD}$ ) for seven animals of proven fertility.

Numbers in parentheses show ranges. 
Table 2. Red wolf sperm abnormalities observed by light microscopy*

\begin{tabular}{|c|c|c|c|}
\hline Abnormality & & $\begin{array}{l}\text { Percentage abnormal } \\
\text { spermatozoa } \\
\text { (mean } \pm \mathrm{SD} \text { ) }\end{array}$ & $\begin{array}{c}\text { Percentage abnormal } \\
\text { spermatozoa } \\
\text { (range) }\end{array}$ \\
\hline \multirow[t]{5}{*}{ Primary } & Acrosome & $0.05 \pm 0.20$ & $0-1$ \\
\hline & Bicephalic & $3.60 \pm 2.80$ & $0-11$ \\
\hline & Microcephalic & $1.40 \pm 2.30$ & $0-8$ \\
\hline & Biflagellate & $0.20 \pm 0.70$ & $0-4$ \\
\hline & Macrocephalic & $0.20 \pm 0.40$ & $0-2$ \\
\hline \multirow[t]{3}{*}{ Secondary } & Coiled & $4.80 \pm 5.20$ & $1-20$ \\
\hline & Detached & $4.80 \pm 4.80$ & $1-29$ \\
\hline & Immature & $4.60 \pm 3.10$ & $1-12$ \\
\hline Tertiary & Bent (neck or tail) & $15.80 \pm 8.20$ & $6-32$ \\
\hline Total & & $35.40 \pm 12.90$ & $13-61$ \\
\hline
\end{tabular}

*Phase-contrast light microscopy at $\times 400$ magnification based on 31 ejaculates.

\section{Electron microscope observations}

Electron microscope observations were carried out on the 31 specimens used in the semen analysis. Nineteen specimens showed abnormal spermatozoa, 18 showed the presence of white blood cells and 11 of these exhibited sperm phagocytosis (spermophagy). Of the 18 sperm samples that were positive for white cells in the electron microscope, nine were also positive in the light microscope analysis carried out at collection, four were negative in the light microscope and five were not evaluated for white cells at the light microscope level.

The fine structure of red wolf spermatozoa is typical of that of other canids, having an oval head, flattened in one plane, modestly sized acrosome and flagellum with typical mid- and principal-piece specializations, a uniformly dense nucleus and closely applied plasma membrane (Fig. 1a).

Abnormalities frequently observed in the thin sections included those associated with head structures, such as incompletely condensed or vacuolated nuclei (not shown), acrosomal defects exemplified by distorted or vacuolated organelles (Fig. 1b) and double-headed spermatozoa (Fig. 1c). Flagellar deformities were also frequently observed in the thin sections. These included coiled axonemes sometimes with hairpin turns proximally back toward the head (not shown), double axonemes either enclosed in the same plasma membrane (Fig. 1d) or with separate flagellar membranes, and bent forms, with the lesion typically occurring at the neck region (Fig. 1e). Bizarre forms showing a multitude of nuclear and cytoplasmic deformities probably of developmental origin (primary abnormalities) were observed on a qualitatively less frequent basis (Fig. 1f).

The most common abnormalities seen under the electron microscope in order of abundance were: double-headed spermatozoa, bent flagellae, acrosomal defects and bizarre forms. These observations cannot be quantitatively compared with the light microscope scoring results for morphologically abnormal spermatozoa (Table 2) because of the restricted sampling constraints of electron microscopy in this study.

\section{Discussion}

The basic semen parameters presented here for red wolves are low compared with similar data for other canids. Mean values for percentage motility of $70-76 \%$, depending on sample selection, were observed in the present study compared with $86 \%$ for the silver fox, Vulpes vulpes (Jalkanen, 1993 ) and $89 \%$ for several other breeds of dog (England and Allen, 1989). The range of motility $(10-95 \%)$ in the present study was greater than that reported for foxes and dogs (60-95\%) (England and Allen, 1989; Jalkanen, 1993). England and Allen (1989) and Jalkanen (1983) used digitally obtained ejaculates rather than samples obtained by electroejaculation as used in the present study. Therefore, stress induced by electroejaculation could be the cause of some of these quantitative differences. In addition, a small number of very poor samples could easily skew the results toward the lower values reported here. Lower figures were also recorded for sperm density, with mean values of $131 \times 10^{6} \mathrm{ml}^{-1}$ for red wolves compared with $322 \times 10^{6} \mathrm{ml}^{-1}$ for foxes (Jalkanen, 1993 ) and $300 \times 10^{6} \mathrm{ml}^{-1}$ for dogs (England and Allen, 1989). The range in sperm density in the study of England and Allen (1989) $\left(60-550 \times 10^{6} \mathrm{ml}^{-1}\right)$ is similar to that observed in the present work $\left(11-560 \times 10^{6} \mathrm{ml}^{-1}\right)$. The total number of spermatozoa for red wolves $\left(470 \times 10^{6}\right)$ is about half that observed in dogs $\left(860 \times 10^{6}\right)$ (Morton and Bruce, 1989) but is similar to that reported by Jalkanen (1993) for foxes $(322 \times$ $\left.10^{6}\right)$.

The percentage of morphologically abnormal spermatozoa was significantly higher for red wolves ( $35 \%$ ) compared with other canids, including foxes (12.5\%) (Jalkanen, 1993) and dogs (11.6\%) (England and Allen, 1989). Morton and Bruce (1989) and Oettle (1993) reported higher percentages of abnormal spermatozoa in dogs of 24 and $21 \%$, respectively. However, the percentage of abnormal spermatozoa reported here for red wolves appears to be at the extreme of the range for canids. Oettle (1993) determined that if the percentage of normal spermatozoa in dogs falls below $60 \%$, there is a precipitous decline in fertility. With the exception of this study, there appears to be little correlation 
(a)

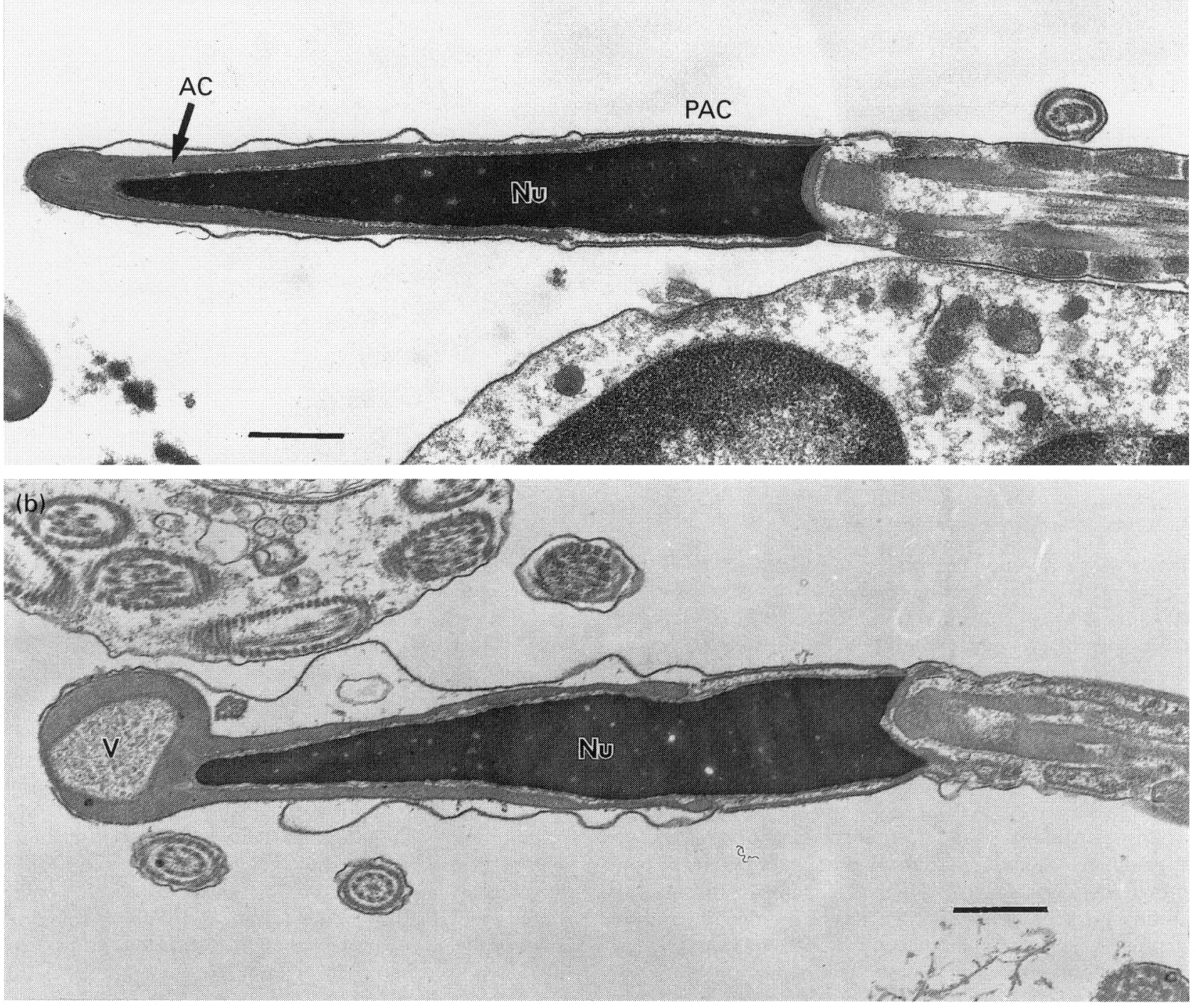

(c)

)

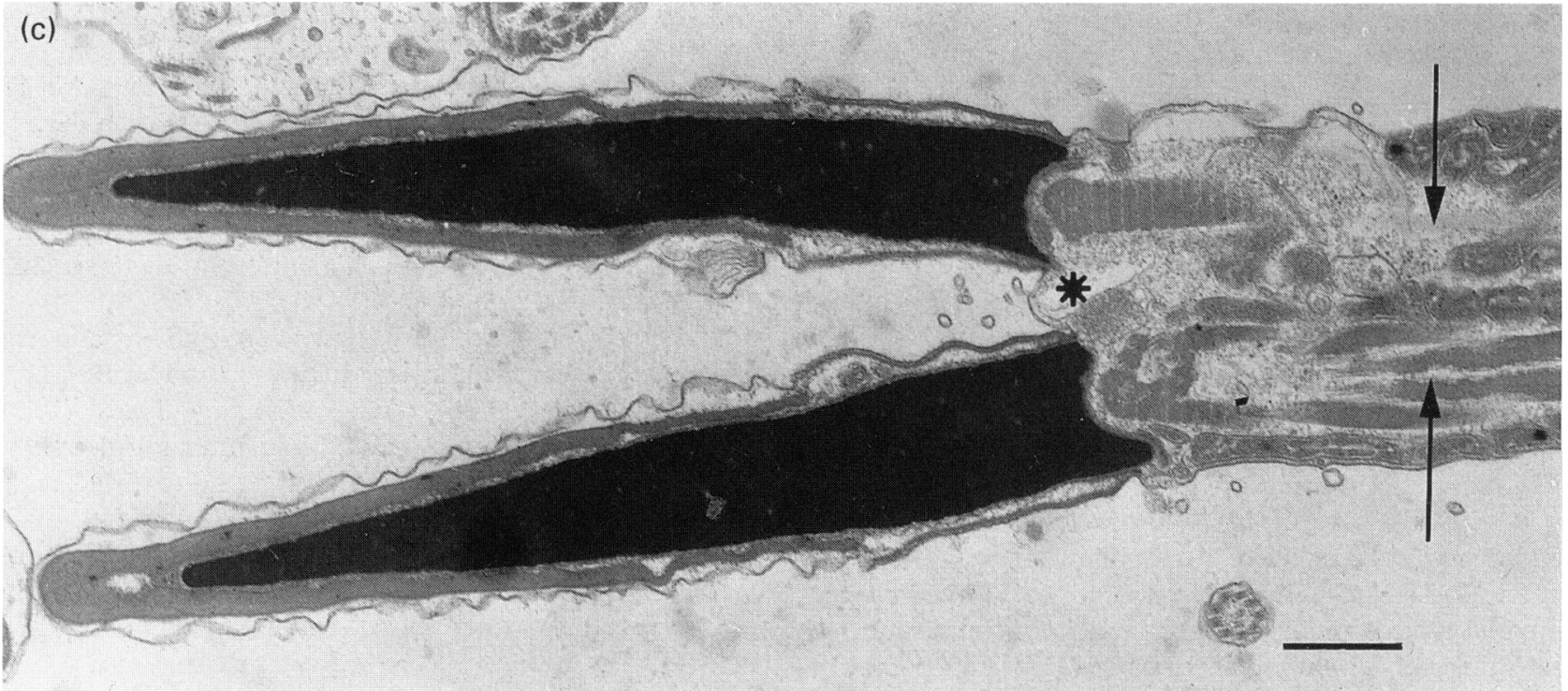

Fig. 1. For legend see p. 100. 


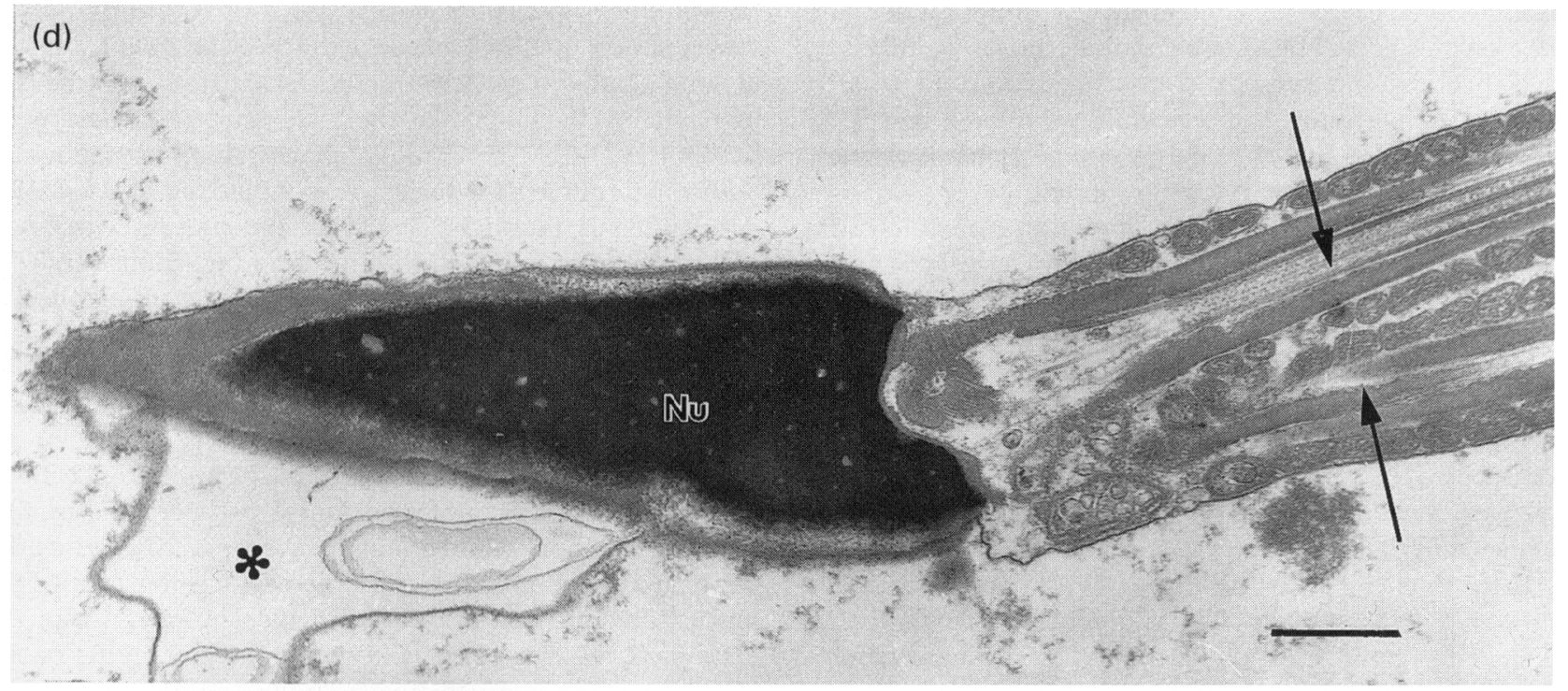

(e)
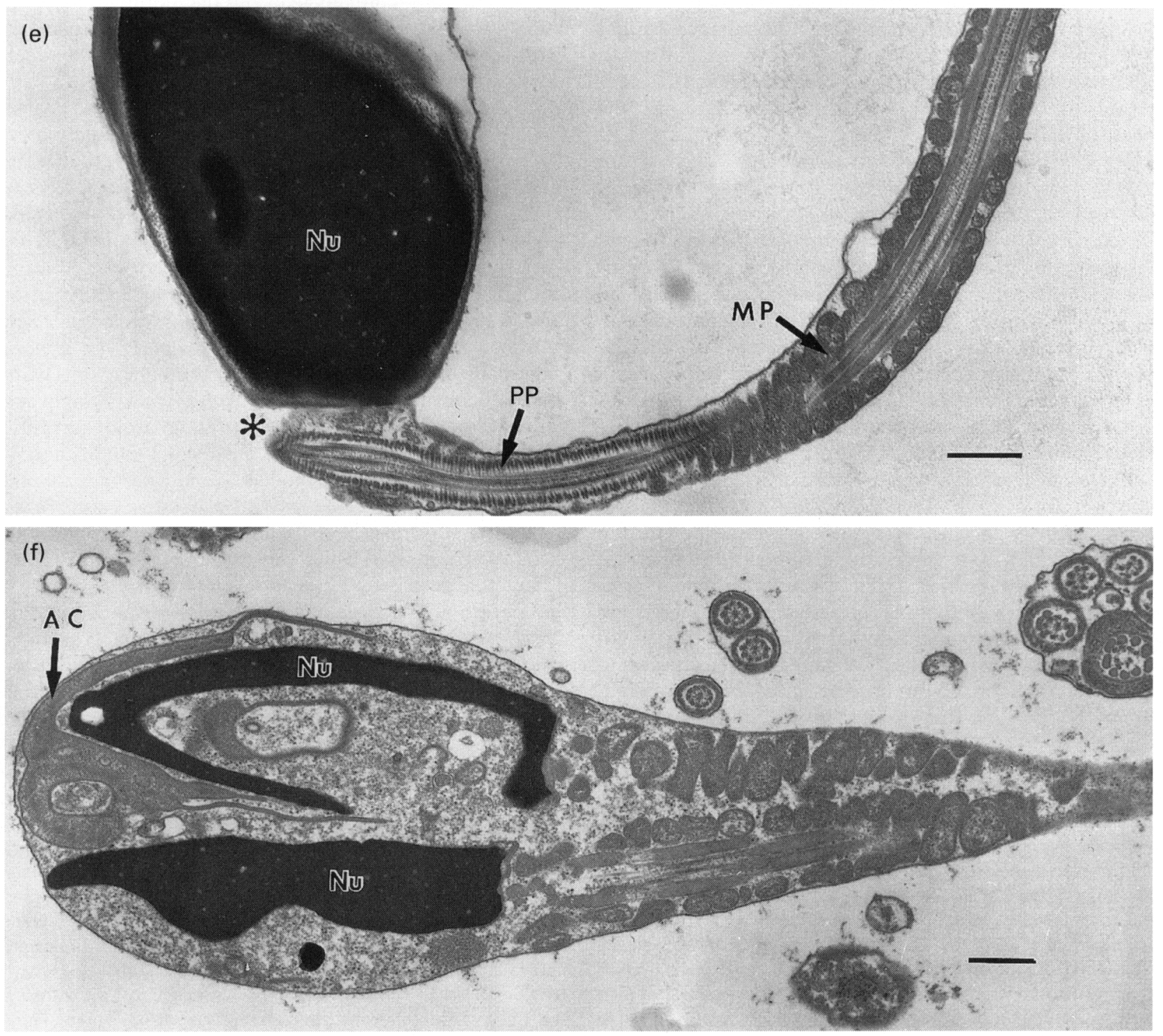

Fig. 1. For legend see p. 100. 
between semen parameters and fertility in canines within the wide variations considered to be normal (England and Allen, 1989). Even if the present data is restricted to proven breeders, the percentage of abnormal spermatozoa is $33 \%$, which supports the theory that this parameter is not a critical determinant in predicting breeding success.

Some qualitative comparisons can be made between the light microscopic categorization of morphologically abnormal spermatozoa and those observed under the electron microscope. Bicephalic spermatozoa and bent and immature forms were frequently observed under the electron microscope and accounted for about two-thirds of the total number of abnormal forms scored under the light microscope. Acrosomal defects and biflagellate forms, also commonly encountered in the electron microscope, were essentially undetected under the light microscope, possibly due to the finer resolution needed to detect these more subtle abnormalities. Conversely, detached (head from tail) and coiled forms comprised almost one-third of abnormalities detected under the light microscope but were rarely seen in the electron microscope. Some of these discrepancies might be due to the fact that thin sections typically comprise only a small portion of a spermatozoon, which does not allow easy determination of gross cell morphology, such as the connection between head and tail.

Many of the extensive catalogue of dog sperm abnormalities described in the work of Oettle and Soley (1988) were also seen in the electron microscope observations reported here. The majority of abnormalities scored by light microscopy, about $85 \%$ of the total, were secondary or tertiary in nature rather than primary (developmental) errors, which is in agreement with the findings of England and Allen (1989) and Jalkanen (1993). Electron microscope studies of cheetah spermatozoa (Gray et al., 1992) showed a high incidence of morphological abnormalities in this species and indicated that the most common defects were coiled or bent tails and axonemal defects, approximately 50 and $30 \%$ of the total, respectively, which is similar to the findings of the present study.

It is unclear why the semen parameters for red wolves are less robust than those of other canids. Almost all comparable studies with canids used digital manipulation to extract spermatozoa rather than electroejaculation. There are obvious reasons for not using digital manipulation with wild carnivores. However, the effects of capture, anaesthesia and electroejaculation on sperm quality must be considered. There is clearly some stress placed on the animals by these procedures. What is not clear is how this would translate to lower sperm quality because, extrapolating data from dogs (Oettle and Soley, 1988), the ejaculated spermatozoa presumably developed in the testis during the preceding 55-70 days. Cold or osmotic shock have been shown to induce morphological defects in spermatozoa (Watson, 1981; England and Plummer, 1993). In the present work such effects were minimized by maintaining fluids at $37^{\circ} \mathrm{C}$, evaluating sperm parameters a few minutes after collection and using isotonic diluents (PBS).

Although efforts were made to avoid using samples that were obviously contaminated with urine, it is possible that a small number of samples were contaminated with urine and these contributed to the low end of the ranges seen in the data. Six of the 31 samples examined had very low motility (below $50 \%$ ), and two had motility values of $10 \%$ or less. The latter two were noted at the time of collection as possible urine contaminated samples and were removed from the data pool; the other low values were retained in the pool. If all six low values are deleted from the motility data, a value of $78 \%$ is achieved compared with $70 \%$ for the inclusive data. This selective higher value for motility is still approximately $10 \%$ lower than that reported for other canids.

It has been suggested that various pathological problems cause increased numbers of abnormal spermatozoa, including insect bites or stings on the scrotum (Bane, 1970), scrotal oedema (Oettle and Soley, 1986) and infections such as Brucella canis (Christiansen, 1984). None of these is likely to be a source of a common problem in a moderately large group of animals. In addition, in the present study the gonads were carefully examined for normal features prior to use. Christiansen (1984) reported that prolonged sexual abstinence results in a massive increase in abnormal spermatozoa in dogs. In this population of wolves, ejaculation was scheduled on a regular (weekly or biweekly) basis during the breeding season with no prolonged periods of abstinence. However, brief abstinent periods cannot be ruled out as a source of some abnormal spermatozoa in the present work.

It is possible that the high degree of pleiomorphism in red wolf spermatozoa is a normal phenomenon that is characteristic of the species and is not a detrimental factor in propagation. There are a few examples of species that have large numbers of morphologically abnormal spermatozoa and propagate effectively, including cheetahs (Wildt et al., 1983) and primates such as humans and gorillas (Afzelius, 1981). However, the great majority of animal species studied

Fig. 1. (a) Morphologically normal red wolf spermatozoon sectioned sagittally through the head region. The acrosome is dense and compact, as is the nuclear material. The post-acrosomal region is well defined and the neck region of the flagellum is straight with normal axonemal components. A non-specifically adhered white cell is visible in the bottom right of the figure. (b) A large acrosomal vesicle is present in this red wolf spermatozoon which also shows extensive plasma membrane blebs. The latter may be artifacts of fixation rather than inherent abnormalities. Other structures appear normal. (c) A double-headed red wolf spermatozoon joined at the neck region (*) that also has dual flagellar components including midpiece mitochondria (arrows). Although all these components are duplicated, specific organelle morphology appears normal. (d) A double flagellar red wolf spermatozoon showing complete duplication of axonemal components (arrows), mitochondria and other tail structures. A large bleb $\left(^{*}\right.$ ) extending from the head plasma membrane is also visible. (e) A red wolf spermatozoon with a bent flagellum with the lesion at the neck region $\left(^{*}\right)$. Note also that a portion of the principal piece has coiled back on itself to invade the space normally reserved for the midpiece. (f) A developmentally defective red wolf spermatid with considerable remaining cytoplasm, two grossly distorted nuclear profiles and a vestigial acrosome. An aborted attempt at midpiece mitochondrial organization is also visible. AC, acrosome; $\mathrm{MP}$, midpiece; $\mathrm{Nu}$, nuclear material; PAC, post-acrosomal region; PP, principal piece; $V$, acrosomal vesicle. Scale bars represent $0.5 \mu \mathrm{m}$. 
exhibit very small percentages of morphologically abnormal spermatozoa.

An earlier study of red wolf semen (Koehler et al., 1994) reported that a significant number of samples showed evidence of white cell infiltration and spermophagy (white cell phagocytosis of spermatozoa). These observations were confirmed in the present study in which white cell infiltration was observed in 18 of the 31 specimens and 11 of these also showed spermophagy. In humans pyospermia and spermophagy have been correlated with decreased fertility (Berger et al., 1982). It is possible that there is a similar correlation in red wolves. However, evidence to support this is not available.

Wildt et al. (1983) suggested that genetic inbreeding may cause large numbers of abnormal spermatozoa $(>50 \%)$ in cheetahs. The population of red wolves used in the present work is descended from a potential founder group of 14 animals. However, the effective founder number was estimated to be eight animals based on genetic restriction considerations (US Fish and Wildlife Service, 1990). It was also estimated that 540 animals ( 320 captive, 220 wild) are required to maintain $80-85 \%$ of the original heterozygosity of the wild stock. Only just over half these numbers have been generated, suggesting a further degradation in heterozygosity percentage. Therefore, gene pool restriction and genetic inbreeding in the remaining small population of red wolves may be a factor in the observed degradation in sperm quality. A detailed genetic analysis of animals correlated with their semen parameters would be a worthwhile extension of these studies.

This research was partially supported by a grant to J. K. Koehler from the Center for Wildlife Conservation. The authors also thank Dale Peterson for his work in the maintenance of the red wolf population at the Graham, Washington (USA) facility.

\section{References}

Afzelius BA (1981) Abnormal human spermatozoa including comparative data from apes American fournal of Primatology 1 175-182
Bane A (1970) The ultrastructure of abnormal spermatozoa in a stud dog Journal of Reproduction and Fertility 22 561-566

Berger RE, Karp LE, Williamson RA, Koehler JK, Moore DE and Holmes KK (1982) The relationship of pyospermia and seminal fluid bacteriology to sperm function as reflected in the sperm penetration assay Fertility and Sterility 37 557-564

Christiansen IJ (1984) Andrology of the normal male. In Reproduction in the Dog and the Cat pp 80-109 Ed. IJ Christiansen. Bailliere Tindall, London

England GCW and Allen WE (1989) Seminal characteristics and fertility in dogs Veterinary Record 125399

England GCW and Plummer JM (1993) Hypo-osmotic swelling of dog spermatozoa Journal of Reproduction and Fertility Supplement 47 261-270

Gray LD, Simmons RS, Schmid-Thedford L, Holiday DB and Marsh C (1992) Defective sperm cells in two captive cheetahs: analysis by TEM and SEM. In Proceedings of the Electron Microscopy Society of America pp 672-673 Eds GW Bailey, J Bentley and JA Small. San Francisco Press Inc., San Francisco

Jalkanen L (1993) Sperm abnormalities in the silver fox (Vulpes vulpes) semen selected for artificial insemination lournal of Reproduction and Fertility Supplement 47 287-290

Koehler JK, Platz CC, Jr, Waddell W, Jones MH, Smith R and Behrns S (1994) Spermophagy in semen of the red wolf, Canis rufus. Molecular Reproduction and Development $37457-461$

Morton DB and Bruce SG (1989) Semen evaluation, cryopreservation and factors relevant to the use of frozen semen in dog Journal of Reproduction and Fertility Supplement $39311-316$

Oettle EE (1993) Sperm morphology and fertility in the dog Jounal of Reproduction and Fertility Supplement 47 257-260

Oettle EE and Soley JT (1986) Severe sperm abnormalities with subsequent recovery following on scrotal oedema and posthitis in a bulldog lournal of Small Animal Practice 27 477-484

Oettle EE and Soley JT (1988) Sperm abnormalities in the dog: a light and electron microscopic study Veterinary Medicine Review 59 28-70

Platz CC and Seager SWJ (1978) Semen collection by electroejaculation in the domestic cat Journal of the American Veterinary Medical Association 1731353 1355

Shaw JH and Jordan PA (1977) The wolf that lost its genes Natural History 86 80-88

US Fish and Wildlife Service (1990) Red Wolf Recovery/Species Survival Plan 110 pp Atlanta, GA, USA

Waddell WT and Behrns SK (1996) Red Wolf (Canis rufus) 1995 International Stud Book 106 pp Point Defiance Zoo and Aquarium

Watson PF (1981) The effects of cold shock on sperm cell membranes. In Effects of Low Temperatures on Biological Membranes pp 187-218 Eds GJ Morris and A Clarke. Academic Press, London

Wildt DE, Bush M, Howard JG, $\mathbf{O}^{\prime}$ Brien SJ, Meltzer D, Van Dyk A, Ebedes $\mathbf{H}$ and Brand DJ (1983) Unique seminal quality in the South African cheetah and a comparative evaluation in the domestic cat Biology of Reproduction 29 1019-1025 